\title{
Optimality Conditions for $\varepsilon$-Vector Equilibrium Problems
}

\author{
Lu Wei-zhong, ${ }^{1}$ Huang Shou-jun,, ${ }^{1,2}$ and Yang Jun ${ }^{1,2}$ \\ ${ }^{1}$ School of Economics and Business Administration, Chongqing University, Chongqing 400030, China \\ ${ }^{2}$ Research Institute of Energy Technology and Economics, Chongqing University, Chongqing 400030, China \\ Correspondence should be addressed to Huang Shou-jun; huangshoujun@cqu.edu.cn
}

Received 20 November 2013; Accepted 2 January 2014; Published 17 February 2014

Academic Editor: Guanglu Zhou

Copyright (C) $2014 \mathrm{Lu}$ Wei-zhong et al. This is an open access article distributed under the Creative Commons Attribution License, which permits unrestricted use, distribution, and reproduction in any medium, provided the original work is properly cited.

By virtue of the separation theorem of convex sets, a necessary condition and a sufficient condition for $\varepsilon$-vector equilibrium problem with constraints are obtained. Then, by using the Gerstewitz nonconvex separation functional, a necessary and sufficient condition for $\varepsilon$-vector equilibrium problem without constraints is obtained.

\section{Introduction}

As the unified model of vector optimization problems, vector variational inequality problems, variational inclusion problems, and vector complementarity problems, vector equilibrium problems have been intensively studied. The existence results for various kinds of vector equilibrium problems have been established, for example, see [1-5] and the references therein. But so far, there are few papers which deal with the properties of the solutions for vector equilibrium problems. Giannessi [3] obtained sufficient conditions for efficient solution and weakly efficient solution to the vector variational inequalities in finite dimensional spaces. Gong [6] obtained some optimality conditions for weakly efficient solution, Henig efficient solution, globally efficient solution, and superefficient solution to vector equilibrium problems with constraints by using the separation theorem of convex sets. Gong [7] the scalarization results for weakly efficient solutions, Henig efficient solutions, and globally efficient solutions to some vector equilibrium problems without constraints.

On the other hand, in some situations, it may not be possible to find an exact solution for an optimization problem, or such an exact solution simply does not exist, for example, if the feasible set is not compact. Thus, it is meaningful to look for an approximate solution instead. There are also many papers to investigate the approximate solution problem, such as [1,8-11]. Kimura et al. [1] obtained several existence results for $\varepsilon$-vector equilibrium problem and the lower semicontinuity of the solution mapping of $\varepsilon$-vector equilibrium problem. Anh and Khanh [10] have considered two kinds of solution sets to parametric generalized $\varepsilon$-vector quasiequilibrium problems and established the sufficient conditions for the Hausdorff semicontinuity (or Berge semicontinuity) of these solution mappings. Gupta and Mehra [9] introduced two new concepts of approximate saddle points and investigated two types of approximate solutions for a vector optimization problem in Banach space setting. X. B. $\mathrm{Li}$ and S. J. Li [11] obtained the Berge lower semicontinuity and Berge continuity of a approximate solution mapping for a parametric vector equilibrium problem.

The aim of this paper is to characterize optimality conditions for $\varepsilon$-vector equilibrium problems. The paper is organized as follows. In Section 2, we recall the main notions and definitions. In Section 3, we obtain some optimality conditions for $\varepsilon$-vector equilibrium problems and $\varepsilon$-vector equilibrium problems with constraints, respectively.

\section{Preliminaries}

Let $X$ and $Z$ be two real Hausdorff topological vector spaces and $V$ be a real locally convex Hausdorff topological vector space. Assume that $S$ and $C$ are two pointed closed convex cones in $V$ and $Z$ with nonempty interior int $S \neq \emptyset$ and int $C \neq \emptyset$, respectively. Let $V^{*}$ and $Z^{*}$ be the topological dual 
space of $V$ and $Z$. Denote the dual cone of $S$ by $S^{*}$ and $C$ by $C^{*}$ :

$$
\begin{aligned}
S^{*} & :=\left\{s^{*} \in V^{*}: s^{*}(s) \geq 0, \forall s \in S\right\}, \\
C^{*} & :=\left\{c^{*} \in Z^{*}: c^{*}(c) \geq 0, \forall c \in C\right\} .
\end{aligned}
$$

Letting $e \in \operatorname{int} S$ be given, we have that $B_{e}^{*}:=\left\{s^{*} \in S^{*}\right.$ : $\left.s^{*}(e)=1\right\}$ is a weak ${ }^{*}$ compact base of $S^{*}$.

Definition 1. Let $E$ be a nonempty convex subset of $X$, and let $f: E \rightarrow V$ be a vector-valued mapping. $f$ is said to be $S$-convex if and only if, for all $y_{1}, y_{2} \in E$ and $l \in[0,1]$,

$$
l f\left(y_{1}\right)+(1-l) f\left(y_{2}\right) \in f\left(l y_{1}+(1-l) y_{2}\right)+S .
$$

Definition 2 (see [12]). Given $e \in \operatorname{int} S$, the Gerstewitz nonconvex separation function $\xi_{e}: V \rightarrow R$ is defined by

$$
\xi_{e}(z)=\min \{t \in R: z \in t e-S\} .
$$

Next, we give some useful properties of the above scalarization functions.

Lemma 3 (see [13]). Let $e \in \operatorname{int} S$. The following properties hold:

(i) $\xi_{e}(z)<r \Leftrightarrow z \in r e-\operatorname{int} S$;

(ii) $\xi_{e a}(z) \geq r \Leftrightarrow z \notin a+r e-\operatorname{int} S$;

(iii) $\xi_{e}(\cdot)$ is a continuous function;

(iv) $\xi_{e}$ is strictly monotonically increasing, that is, if $z_{1}-z_{2} \in$ int $S \Rightarrow \xi_{e}\left(z_{1}\right)>\xi_{e}\left(z_{2}\right)$.

\section{Optimality Conditions}

In this section, we first deal with the following $\varepsilon$-vector equilibrium problem with constraints (for short $\varepsilon$-VEPC): find $\bar{x} \in K$ such that

$$
f(\bar{x}, y)+\varepsilon e \notin-\operatorname{int} S, \quad \forall y \in K,
$$

and the constraint set

$$
K=\{x \in E: g(x) \in-C\},
$$

where $E$ is a nonempty subset of $X, f: E \times E \rightarrow V$ is a vectorvalued mapping, $g: E \rightarrow Z$ is a vector-valued mapping, $e \in \operatorname{int} S$, and $\varepsilon$ is a positive real number.

If $f(x, y)=g(y)-g(x), x, y \in K$, and if $\bar{x} \in K$ is a solution of $\varepsilon$-VEP, then $\bar{x} \in K$ is a solution of $\varepsilon$-efficient solution of vector optimization problem of $g$, where $g$ is a vector-valued mapping.

First, we give some necessary and sufficient conditions for $\varepsilon$-vector equilibrium problem with constraints by using the separation theorem of convex sets.

(H) Convexity Assumption: for all $y_{1}, y_{2} \in E$ and $l \in$ $[0,1]$, there exists $\bar{y} \in E$ such that

$$
\begin{gathered}
l f\left(x, y_{1}\right)+(1-l) f\left(x, y_{2}\right) \in f(x, \bar{y})+S, \quad \forall x \in E, \\
\lg \left(y_{1}\right)+(1-l) g\left(y_{2}\right) \in g(\bar{y})+C .
\end{gathered}
$$

Remark 4. (i) Assumption (H) does not require that $E$ be a convex set.

(ii) We say that $f$ is $S$-convex-like in $y$ if $f$ satisfies (6) and that $g$ is $C$-convex-like if $g$ satisfies (7).

(iii) If $E$ is a convex set, $f$ is $S$-convex in $y$, and $g$ is $C$ convex, then Assumption ( $\mathrm{H}$ ) is satisfied.

Theorem 5. Lete $\in \operatorname{int} S$ and $E$ be a nonempty subset of $X$. Let $f: E \times E \rightarrow V$ be a vector-valued mapping with $f(x, x)=0$, for all $x \in E$ and let $g: E \rightarrow Z$ be a vector-valued mapping. Assume that $(H)$ is satisfied and there exists $x_{0} \in E$ such that $g\left(x_{0}\right) \in-\operatorname{int} C$. If $\bar{x} \in K$ is a solution of $\varepsilon$-VEPC, then there exists $s^{*} \in B_{e}^{*}$ and $c^{*} \in C^{*}$ such that

$$
\begin{gathered}
s^{*}(f(\bar{x}, y))+c^{*}(g(\bar{x})) \geq-\varepsilon, \quad \forall y \in E \\
0 \geq c^{*}(g(\bar{x})) \geq-\varepsilon,
\end{gathered}
$$

where $\varepsilon$ is a positive real number.

Proof. Let $\bar{x} \in K$ be a solution of $\varepsilon$-VEPC. We consider the set

$$
\begin{gathered}
M_{\bar{x}}=\left\{(y, z) \in V \times Z: y-f\left(\bar{x}, y^{\prime}\right)-\varepsilon e \in \operatorname{int} S,\right. \\
\left.z-g\left(y^{\prime}\right) \in \operatorname{int} C, \text { for some } y^{\prime} \in E\right\} .
\end{gathered}
$$

By assumptions, it is clear that $M_{\bar{x}} \neq \emptyset$ and $M_{\bar{x}}$ is an open set. Now, we show that $\left(0_{V}, 0_{Z}\right) \notin M_{\bar{x}}$. If not, by the definition of $M_{\bar{x}}$, then there exists $y_{0} \in E$ such that

$$
f\left(\bar{x}, y_{0}\right)+\varepsilon e \epsilon-\operatorname{int} S, \quad g\left(y_{0}\right) \in-\operatorname{int} C .
$$

Thus, $y_{0} \in K$. This contradicts that $\bar{x}$ is a solution of $\varepsilon$-VEPC. Hence, $\left(0_{V}, 0_{Z}\right) \notin M_{\bar{x}}$.

Next, we show that $M_{\bar{x}}$ is a convex set. Let $\left(y_{1}, z_{1}\right)$, $\left(y_{2}, z_{2}\right) \in M_{\bar{x}}$ and $l \in[0,1]$. By the definition of $M_{\bar{x}}$, there exist $y_{1}^{\prime}, y_{2}^{\prime} \in E$ such that

$$
\begin{array}{cc}
y_{1}-f\left(\bar{x}, y_{1}^{\prime}\right)-\varepsilon e \in \operatorname{int} S, & y_{2}-f\left(\bar{x}, y_{2}^{\prime}\right)-\varepsilon e \in \operatorname{int} S, \\
z_{1}-g\left(y_{1}^{\prime}\right) \in \operatorname{int} C, & z_{2}-g\left(y_{2}^{\prime}\right) \in \operatorname{int} C .
\end{array}
$$

Then, by (11), we have

$$
\begin{gathered}
l y_{1}+(1-l) y_{2}-\varepsilon e-\left(l f\left(\bar{x}, y_{1}^{\prime}\right)+(1-l) f\left(\bar{x}, y_{2}^{\prime}\right)\right) \in \operatorname{int} S, \\
l z_{1}+(1-l) z_{2}-\left(l g\left(y_{1}^{\prime}+(1-l) g\left(y_{2}^{\prime}\right)\right)\right) \in \operatorname{int} C .
\end{gathered}
$$

By assumptions, there exists $\bar{y} \in E$ satisfying $l y_{1}+(1-l) y_{2}-$ $\varepsilon e-f(\bar{x}, \bar{y}) \in \operatorname{int} S$ and $l z_{1}+(1-l) z_{2}-g(\bar{y}) \in \operatorname{int} C$. Namely, $M_{\bar{x}}$ is a convex set.

Thus, by the separation theorem of convex sets, there exists $(0,0) \neq\left(s_{1}^{*}, c_{1}^{*}\right) \in\left(S^{*} \times C^{*}\right)$ such that

$$
s_{1}^{*}(y)+c_{1}^{*}(z)>0, \quad \forall(y, z) \in M_{\bar{x}} .
$$

Let $(y, z) \in M_{\bar{x}}$, there exists $y^{\prime} \in E$ such that $y-f\left(\bar{x}, y^{\prime}\right)-$ $\varepsilon e \in \operatorname{int} S$ and $z-g\left(y^{\prime}\right) \in \operatorname{int} C$. Hence, for every $s \in \operatorname{int} S$, 
$c \in \operatorname{int} C, t_{1}>0$ and $t_{2}>0$, we have $\left(y+t_{1} s, z\right) \in M_{\bar{x}}$ and $\left(y, z+t_{2} c\right) \in M_{\bar{x}}$. By $(13)$,

$$
s_{1}^{*}(y+t s)+c_{1}^{*}(z)>0, \quad \forall s \in \operatorname{int} S, t>0 .
$$

Letting $t \rightarrow \infty$, we get $s_{1}^{*}(s) \geq 0$, for all $s \in$ int $S$. Since $S$ is closed convex cone, by the continuity of $s_{1}^{*}, s_{1}^{*}(s) \geq 0$, for all $s \in S$; that is, $s_{1}^{*} \in S^{*}$. Similarly, $c_{1}^{*} \in C^{*}$. We next show $s_{1}^{*} \neq 0$. In fact, if $s_{1}^{*}=0$, by (13), we have

$$
c_{1}^{*}(z)>0, \quad \forall(y, z) \in M_{\bar{x}} .
$$

By assumptions, there exists $x_{0} \in E$ such that $g\left(x_{0}\right) \in-\operatorname{int} C$. Then, we get $c_{1}^{*}\left(g\left(x_{0}\right)\right) \leq 0$. For the above $x_{0}$, we have

$$
\begin{array}{r}
\left(f\left(\bar{x}, x_{0}\right)+\varepsilon e+s, g\left(x_{0}\right)+c\right) \in M_{\bar{x}}, \\
\forall s \in \operatorname{int} S, c \in \operatorname{int} C .
\end{array}
$$

By $(15), c_{1}^{*}\left(g\left(x_{0}\right)\right)>0$. This is a contradiction. Namely, $s_{1}^{*} \neq 0$.

It is clear that $(f(\bar{x}, \bar{x})+\varepsilon e+t s, g(\bar{x})+t c) \in M_{\bar{x}}$, for all $t>0, s \in \operatorname{int} S$ and $c \in \operatorname{int} C$. Thus, by (13), we have that

$$
s_{1}^{*}(f(\bar{x}, \bar{x})+\varepsilon e+t s)+c_{1}^{*}(g(\bar{x})+t c)>0 .
$$

Letting $t \rightarrow 0$,

$$
c_{1}^{*}(g(\bar{x})) \geq-\varepsilon s_{1}^{*}(e) .
$$

Since $s_{1}^{*}(e)>0, c_{1}^{*} / s_{1}^{*}(e) \in C^{*}$. Thus, letting $c^{*}=c_{1}^{*} / s_{1}^{*}(e)$,

$$
c^{*}(g(\bar{x})) \geq-\varepsilon .
$$

By assumptions, it is clear that $c^{*}(g(\bar{x})) \leq 0$.

Since $(f(\bar{x}, y)+\varepsilon e+t s, g(y)+t c) \in M_{\bar{x}}$, for all $y \in E$, $t>0, s \in \operatorname{int} S$ and $c \in \operatorname{int} C$, by (13) and letting $t \rightarrow 0$, we have

$$
\frac{s_{1}^{*}(f(\bar{x}, y))+c_{1}^{*}(g(y))}{s_{1}^{*}(e)} \geq-\varepsilon, \quad \forall y \in E .
$$

Let $s^{*}=s_{1}^{*} / s_{1}^{*}(e) \in B_{e}^{*}$. Then,

$$
s^{*}(f(\bar{x}, y))+c^{*}(g(\bar{x})) \geq-\varepsilon, \quad \forall y \in E .
$$

This completes the proof.

Theorem 6. Let $e \in \operatorname{int} S$ and let $E$ be a nonempty subset of $X$. Let $f: E \times E \rightarrow V$ be a vector-valued mapping with $f(x, x)=$ 0 , for all $x \in E$ and let $g: E \rightarrow Z$ be a vector-valued mapping. If there exist $\bar{x} \in K, s^{*} \in B_{e}^{*}$, and $c^{*} \in C^{*}$ such that

$$
\begin{gathered}
s^{*}(f(\bar{x}, y))+c^{*}(g(\bar{x})) \geq-\varepsilon, \quad \forall y \in E \\
0 \geq c^{*}(g(\bar{x})) \geq-\varepsilon,
\end{gathered}
$$

then $\bar{x}$ is a solution of $\varepsilon-V E P C$, where $\varepsilon$ is a positive real number.

Proof. Let $\bar{x} \in K$, and suppose that there exist $s^{*} \in B_{e}^{*}$ and $c^{*} \in C^{*}$ such that

$$
\begin{gathered}
s^{*}(f(\bar{x}, y))+c^{*}(g(\bar{x})) \geq-\varepsilon, \quad \forall y \in E, \\
c^{*}(g(\bar{x})) \geq-\varepsilon .
\end{gathered}
$$

We next will show that $\bar{x} \in K$ is a solution of $\varepsilon$-VEPC. If not, then there exists $\bar{y} \in K$ such that

$$
f(\bar{x}, \bar{y})+\varepsilon e \in-\operatorname{int} S .
$$

Since $s^{*} \in B_{e}^{*}$ and $\bar{y} \in K$, we have

$$
s^{*}(f(\bar{x}, \bar{y}))<-\varepsilon, \quad c^{*}(g(\bar{y})) \leq 0 .
$$

Thus, by (23),

$$
-\varepsilon \leq s^{*}(f(\bar{x}, y))+c^{*}(g(\bar{x}))<-\varepsilon+0=-\varepsilon .
$$

This is a contradiction. Hence, $\bar{x} \in K$ is a solution of $\varepsilon$-VEPC. This completes the proof.

Next, we consider the following $\varepsilon$-vector equilibrium problem without constraints (for short $\varepsilon$-VEP): find $\bar{x} \in E$ such that

$$
f(x, y)+\varepsilon e \notin-\operatorname{int} S, \quad \forall y \in E,
$$

where $E$ is a nonempty subset of $X, f: E \times E \rightarrow V$ is a vectorvalued mapping, $e \in \operatorname{int} S$, and $\varepsilon$ is a positive real number.

If $f(x, y)=g(y)-g(x), x, y \in E$, and if $\bar{x} \in E$ is a solution of $\varepsilon$-VEP, then $\bar{x} \in E$ is a solution of $\varepsilon$-efficient solution of vector optimization problem of $g$, where $g$ is a vector-valued mapping.

Theorem 7. Let $e \in \operatorname{int} S$ and $E$ be a nonempty subset of $X$. Let $f: E \times E \rightarrow V$ be a vector-valued mapping with $f(x, x)=0$, for all $x \in E$. Then $\bar{x} \in E$ is a solution of $\varepsilon-V E P$ if and only if

$$
\xi_{e}(f(\bar{x}, y)) \geq-\varepsilon, \quad \forall y \in E,
$$

where $\varepsilon$ is a positive real number.

Proof. If $\bar{x} \in E$ is a solution of $\varepsilon$-VEP, then

$$
(f(\bar{x}, E)+\varepsilon e) \bigcap(-\operatorname{int} S)=\emptyset .
$$

By Lemma 3(ii), we have

$$
\xi_{e}(f(\bar{x}, y)) \geq-\varepsilon, \quad \forall y \in E .
$$

On the other hand, suppose that $\bar{x} \in E$ is not a solution of $\varepsilon$-VEP. Then, there exists $\bar{y} \in E$ such that

$$
f(\bar{x}, \bar{y})+\varepsilon e \in-\operatorname{int} S .
$$

By Lemma 3(i), we have

$$
\xi_{e}(f(\bar{x}, \bar{y}))<-\varepsilon .
$$

This is a contradiction. This completes the proof.

\section{Conflict of Interests}

The authors declare that there is no conflict of interests regarding the publication of this paper. 


\section{Acknowledgments}

This research was supported by Project supported by the Key Program of National Natural Science of China (Grant no. 71133007), National Natural Science Foundation of China (Grant no. 71373297), and Program for New Century Excellent Talents in University of Ministry of Education of China (NCET-10-0883).

\section{References}

[1] K. Kimura, Y. C. Liou, and J. C. Yao, "Semicontinuty of the solution mapping of $\varepsilon$-vector equilibrium problem," in Globalization Challenge and Management Transformation, pp. 103-113, Science Press, Beijing, China, 2007.

[2] G. Y. Chen, "Existence of solutions for a vector variational inequality: an extension of the Hartmann-Stampacchia theorem," Journal of Optimization Theory and Applications, vol. 74, no. 3, pp. 445-456, 1992.

[3] F. Giannessi, Vector Variational Inequalities and Vector Equilibria: Mathematical Theories, Kluwer Academic Publishers, Dordrecht, The Netherlands, 2000.

[4] F. Giannessi, A. Maugeri, and P. M. Pardalos, Equilibrium Problems: Nonsmooth Optimization and Variational Inequality Models, Springer, Berlin, Germany, 2002.

[5] F. Giannessi, Constrained Optimization and Image Space Analysis, Springer, Berlin, Germany, 2005.

[6] X.-H. Gong, "Optimality conditions for vector equilibrium problems," Journal of Mathematical Analysis and Applications, vol. 342, no. 2, pp. 1455-1466, 2008.

[7] X.-H. Gong, "Scalarization and optimality conditions for vector equilibrium problems," Nonlinear Analysis. Theory, Methods \& Applications A, vol. 73, no. 11, pp. 3598-3612, 2010.

[8] K. Kimura and J. C. Yao, "Semicontinuity of solution mappings of parametric generalized vector equilibrium problems," Journal of Optimization Theory and Applications, vol. 138, no. 3, pp. 429443, 2008.

[9] D. Gupta and A. Mehra, "Two types of approximate saddle points," Numerical Functional Analysis and Optimization, vol. 29, no. 5-6, pp. 532-550, 2008.

[10] L. Q. Anh and P. Q. Khanh, "Semicontinuity of the approximate solution sets of multivalued quasiequilibrium problems," Numerical Functional Analysis and Optimization, vol. 29, no. 1-2, pp. 24-42, 2008.

[11] X. B. Li and S. J. Li, "Continuity of approximate solution mappings for parametric equilibrium problems," Journal of Global Optimization Dealing, vol. 51, no. 3, pp. 541-548, 2011.

[12] C. Gerstewitz, "Nichtkonvexe trennungssätze und deren anwendung in der theorie der vektoroptimierung," Seminarberichte der Secktion Mathematik der HumboldtUniversität zu Berlin, vol. 80, pp. 19-31, 1986.

[13] C. Gerth and P. Weidner, "Nonconvex separation theorems and some applications in vector optimization," Journal of Optimization Theory and Applications, vol. 67, no. 2, pp. 297320, 1990 . 


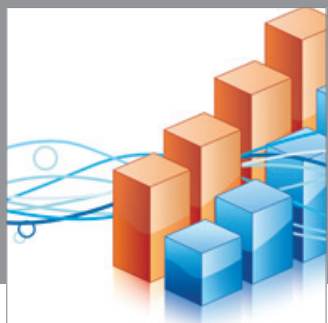

Advances in

Operations Research

mansans

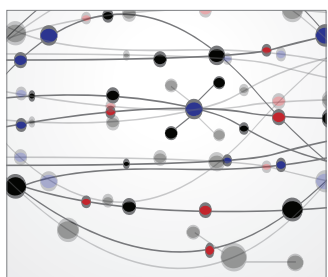

The Scientific World Journal
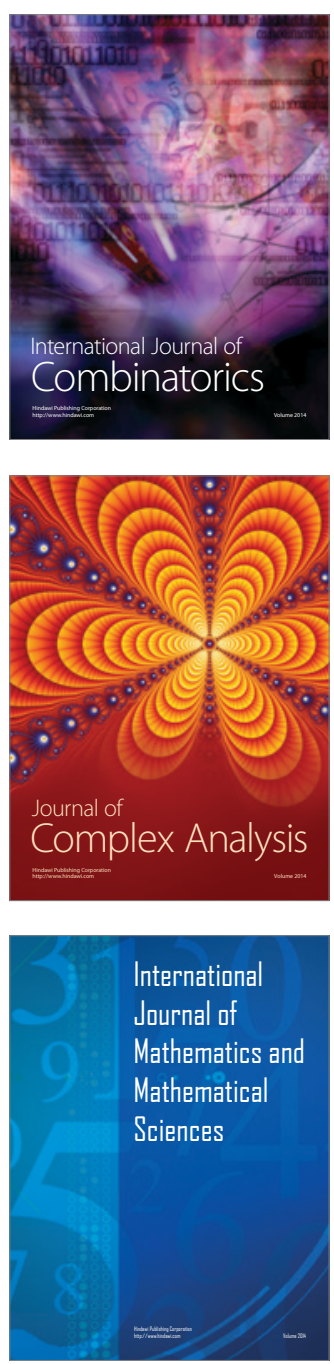
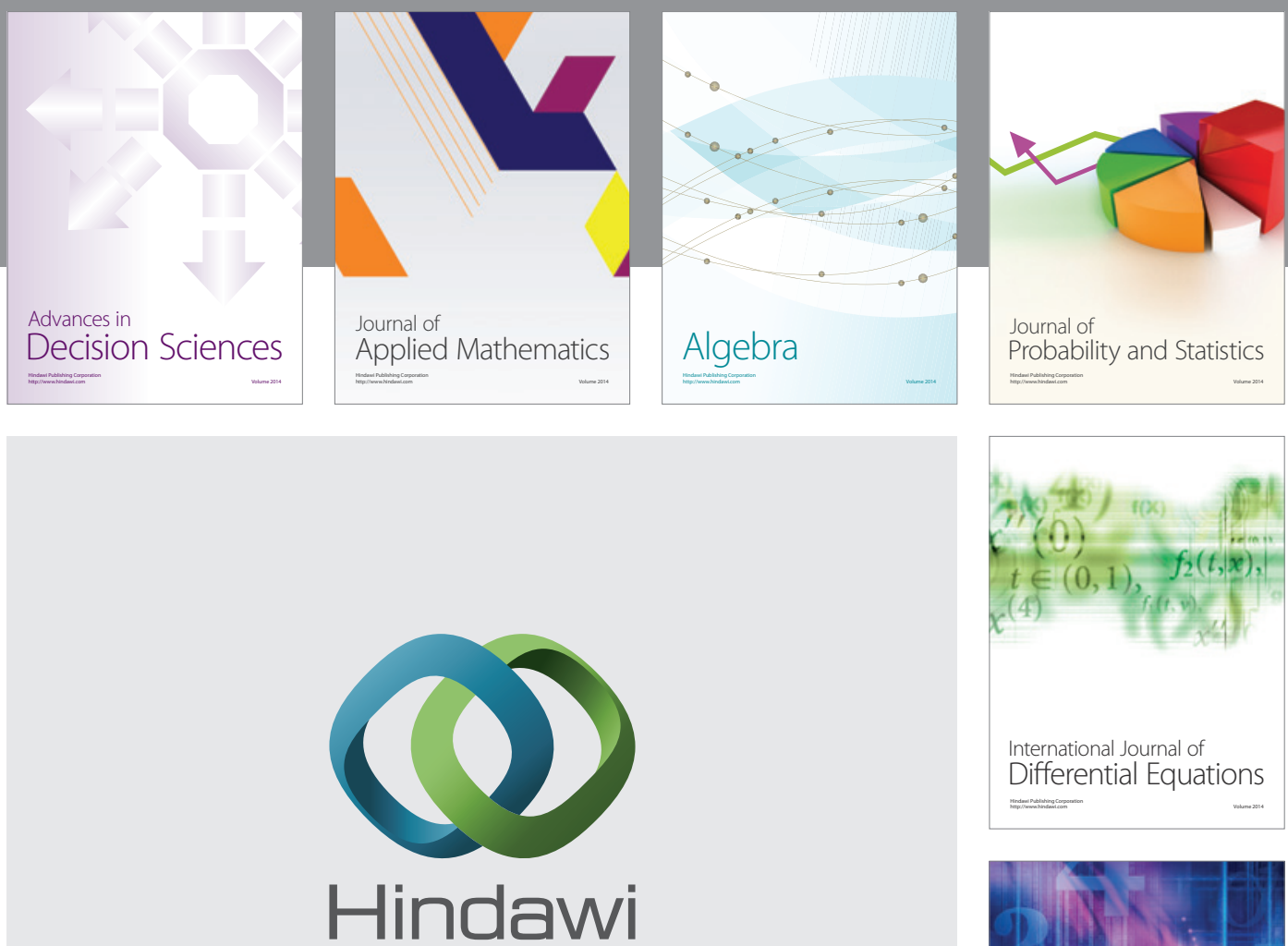

Submit your manuscripts at http://www.hindawi.com
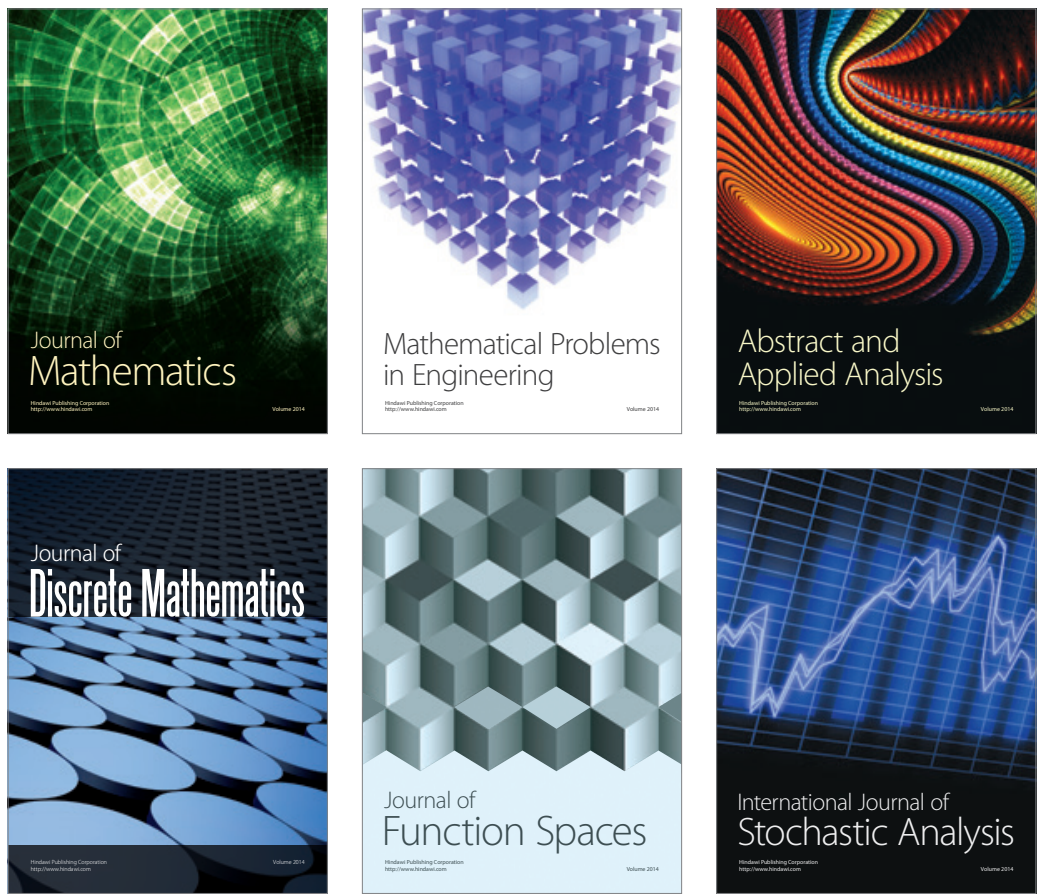

Journal of

Function Spaces

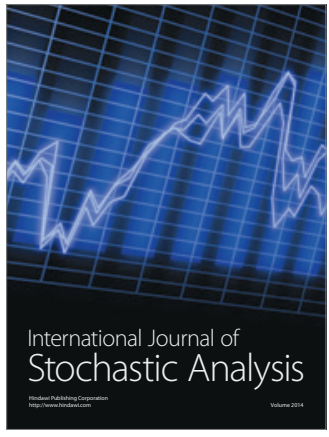

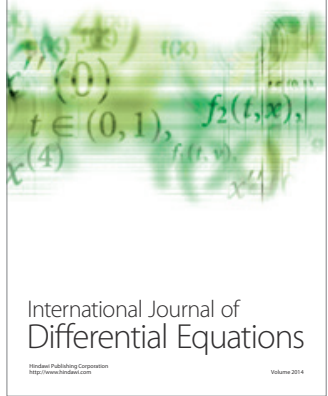
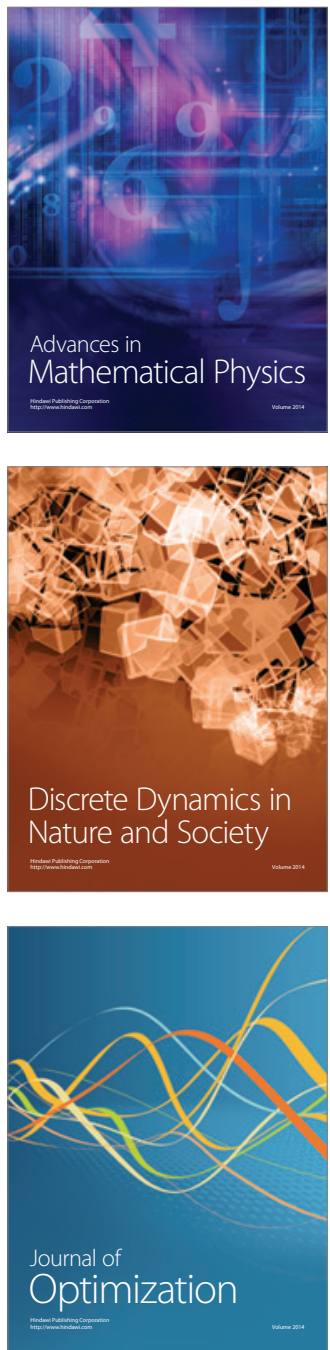